\title{
El cambio climático y sus representaciones sociales en el medio rural de Chiapas,
} México

Climate Change and its Social Representations in the Rural Zones of Chiapas, Mexico

José Antonio Santiago Lastra ${ }^{a}$

Universidad Intercultural de Chiapas, México

jlastra@unich.edu.mx

ORCID: http://orcid.org/0000-0002-4692-0035

Luz Helena Horita Pérez

Universidad Intercultural de Chiapas, México

ORCID: http://orcid.org/0000-0002-5787-0352

José Luis Sulvarán López

Universidad Intercultural de Chiapas, México

ORCID: http://orcid.org/0000-0003-4152-4903
DOI: https://doi.org/10.11144/Javeriana.ayd22-42.ccrs Redalyc: http://www.redalyc.org/articulo.oa? $\mathrm{id}=151557418004$

Fecha de recepción: 20 Julio 2017 Fecha de publicación: 20 Julio 2018

\section{Resumen:}

El objetivo del presente trabajo fue analizar las representaciones sociales de las mujeres y los hombres de las comunidades rurales sobre los cambios ambientales ocurridos en el estado de Chiapas. La investigación se realizó con un enfoque estructural exploratorio de tipo interpretativo, para ello se identificaron sabios de comunidades rurales representativas de las distintas regiones biogeográficas del estado. Se realizó un análisis hermenéutico, se construyó una tipología para la comprensión de las representaciones sociales estudiadas y se verificó la validez de dicha tipología mediante la prueba estadística de Ji cuadrada. Como resultado, se encontró un fuerte consenso sobre la percepción de un aumento en la temperatura y cambios en el patrón de lluvias, lo cual concuerda con los escenarios modelados para el estado, desde la visión de la ciencia occidental.

Palabras clave: cambios ambientales, historias de vida, sabios comunitarios.

\begin{abstract}
:
This work sought to analyze the social representations of women and men in the rural communities about the environmental changes that occurred in the Chiapas State. The research was conducted under an interpretative exploratory structural approach. To do so, the sages belonging to rural communities representative of the different biogeographical regions in the Chiapas State were identified. A hermeneutic analysis was carried out and a typology was built in order to understand the social representations examined therein. The validity of this typology was verified through the chi-square test. As a result, a strong consensus was found regarding the people's perception of an increase in the temperature as well as some changes in the rain pattern, which agrees with the models made by the State based on the western science vision.
\end{abstract}

Keywords: environmental changes, life stories, community sages.

\section{Introducción}

En la actualidad, las transformaciones del medio ambiente, como consecuencia de las actividades humanas bajo un modelo de desarrollo capitalista patriarcal, es decir autoritario, destructivo y excluyente, alteran de manera directa o indirecta la composición de la atmósfera, el agua, el suelo y la biodiversidad, lo que genera una crisis ambiental que alcanza una dimensión planetaria, denominada cambio climático global.

Para construir propuestas ante dicha crisis ambiental, es necesaria una racionalidad más integral, es decir, sistémica, constructiva, incluyente y justa, que tenga en cuenta la interpretación y la construcción de significados de las comunidades rurales sobre sus realidades ambientales. Estas sólo pueden partir de las mediaciones socioculturales, nutridas por la subjetividad individual y colectiva, y construidas a partir de diversas formas de interacción y diferentes temporalidades, de tal forma que las propuestas 
respondan a necesidades locales y regionales del ambiente, y generen iniciativas endógenas que contribuyan, al mismo tiempo, a paliar desde lo local a una situación global que nos compete a todos (Figueredo, 2012).

En este sentido, el presente artículo tiene como objetivo analizar las representaciones sociales (Abric, 2001) de las mujeres y los hombres de las comunidades rurales, que todos los días interactúan de manera cercana con los sistemas naturales para obtener su sustento, sobre los cambios ambientales ocurridos en el estado de Chiapas, mediante el uso de historias de vida y haciendo énfasis en el cambio climático (Tinoco \& Bellato, 2006).

\section{Representaciones sociales}

El concepto de representaciones sociales se ha definido como el conjunto de conocimientos, valores y actitudes generados por el sentido común (Abric, 2001), es decir, es el cómo nos concebimos frente al entorno e interpretamos al entorno, trascendiendo la relación sujeto - objeto y entrelazando al sujeto colectivo.

La Teoría de las Representaciones Sociales, presentada por Moscovici en la década de los sesenta, tiene gran vigencia ya que abrió un espacio de investigación esencial para comprender los fenómenos sociopolíticos y ambientales de interés actual: el estudio del "conocimiento del sentido común”. Acceder a las representaciones sociales a partir de procesos comunicativos nos permite comprender cómo piensan los individuos y cómo organizan su vida cotidiana en las esferas de lo privado y lo público (Gastrón, 2003).

El trabajo realizado por Moscovici se inscribe en el paradigma constructivista, el cual reconoce una realidad aprehensible en forma de múltiples e intangibles constructos conformados mental y socialmente por medio de la experiencia vivida. La ventaja del enfoque de la Teoría de las Representaciones Sociales es que conjuga por igual las dimensiones cognitivas y sociales de la construcción de la realidad. Entre las principales fuentes desde las que se nutren las representaciones sociales, Meira (2013) reconoce, en un primer momento, la información y conocimientos validados desde fuentes científicas, mediáticas y de la tradición cultural. En segundo lugar, ubica todos aquellos procesos de interacción social en los que se comparten, intercambian y reelaboran interpretaciones, adquiriendo así el carácter social. Y, en último término, señala todos los procesos cognitivos de orden individual que permiten valorar, jerarquizar e integrar los nuevos conocimientos. Así, las representaciones sociales proceden de observaciones desde los distintos ámbitos de la experiencia, y se apropian "a diestra y siniestra de nociones y lenguajes de las ciencias o de las filosofías", extrayendo conclusiones de carácter ecléctico (Moscovici, 1979, p.30).

Debido a la pluralidad de elementos que entran en juego para conformar las representaciones sociales, el abordaje para su estudio ha tenido un carácter particularmente interdisciplinar. $\mathrm{Al}$ reconocer desde los estudios antropológicos la noción de cultura propuesta por Geertz, como "una red de significaciones y sentidos que sirve para significar la vida", y la propuesta por García-Castaño, como "totalidad de creencias aprendidas, herramientas y tradiciones compartidas por un grupo con la finalidad de dar continuidad, orden y significado a sus vidas" (Rizo \& Romeu, 2006, p.7), las representaciones sociales están relacionadas directamente con las matrices culturales que participan en el contexto social. Las representaciones sociales se configuran con base en el saber acumulado (en un primer momento de orden cultural) de los participantes y el contexto socioambiental en el que se vinculan.

De esta forma, dichas representaciones conforman sistemas de conocimientos en los que se manifiestan estereotipos, opiniones, creencias, normas y valores circulantes en un contexto socioambiental determinado. El conjunto de elementos señalados da lugar al conocimiento espontáneo, llamado conocimiento del sentido común, constituido por contenidos cognitivos, afectivos y simbólicos, orientadores de las conductas en el ámbito de lo cotidiano. En palabras de Moscovici, las representaciones sociales constituyen "una forma de conocimiento particular de la sociedad, irreductible a cualquier cosa” (Moscovici, 1979, p.30; Ibáñez, 1994; Knapp, Suárez, \& Mesa, 2003). 
El conocimiento constituido desde las representaciones sociales permite la construcción de la realidad desde los factores culturales circundantes mediante los procesos de objetivación y anclaje, y las prácticas sociales de comunicación en las que estamos inmersos. Por un lado, la objetivación permite generar índices y significantes que hacen real un esquema conceptual sobre el objeto o fenómeno natural representado, acortando la separación entre la ciencia y lo real entre el discurso especializado y conceptos previamente naturalizados (Moscovici, 1979). Por otro, el anclaje es el proceso mediante el cual "la sociedad cambia el objeto social por un instrumento del que puede disponer", colocándolo en una escala de preferencia de las relaciones sociales existentes. Así, mientras la objetivación "traslada la ciencia al dominio del ser, el anclaje la delimita en el del hacer" (Moscovici, 1979, p.76).

La consideración dialéctica en la constitución de las representaciones sociales permite entablar un diálogo entre la dimensión de la tradición (lo que ya está dado y nos identifica), y la dimensión de la innovación, de lo que se construye en el quehacer cotidiano. Lo anterior hace evidente la importancia de los procesos comunicativos y los actores que participan en el grupo social, en la configuración y actualización de las representaciones sociales elaboradas en torno a una temática. Así, una vertiente de los estudios de las representaciones sociales es la estructural, que permite identificar elementos centrales de la representación, con cierto grado de permanencia, de aquellos que son periféricos y que varían de acuerdo con características concretas de los participantes. La segunda vertiente de estudios enfatiza la aproximación por medio del estudio de los procesos de construcción de las representaciones sociales. Este enfoque procesual corresponde a la escuela clásica desarrollada por Denise Jodelet, en la cual se privilegia el análisis de lo social, la cultura e interacciones sociales que intervienen en la formación del corpus, con una tendencia al análisis cualitativo de contenidos. El enfoque estructural recurre a técnicas experimentales para estudiar tanto los elementos del núcleo figurativo (elemento central) de las representaciones sociales, como la estructura cognitiva que los aloja. En ambos enfoques se conserva el carácter inter y transdisciplinar para su abordaje.

Por lo tanto, mediante las representaciones sociales es posible aproximarse a la realidad de los individuos ante temas tan complejos como el cambio climático global (Ramírez-Vázquez \& González-Gaudiano, 2016), y para el presente estudio se recurrió al enfoque estructural en el contexto del medio rural.

\section{Cambio climático global}

La acción del ser humano sobre el planeta influye de forma significativa sobre las concentraciones de gases de efecto invernadero (GEI). Desde 1750, las concentraciones de $\mathrm{CO}_{2}$ han aumentado en un 40\%; y en el año 2013, alcanzó un máximo histórico de 396 ppm (Organización Meteorológica Mundial [OMM], 2014). Hoy día se retiene más calor en la atmósfera, lo que aumenta la temperatura media global de la superficie terrestre, con respecto a lo registrado en la época preindustrial, hasta finales del siglo XVIII (Grupo Panel Intergubernamental sobre Cambio Climático [IPCC], 2013).

Según el IPCC, el aumento en la temperatura oscila en los $0,85^{\circ} \mathrm{C}$ durante el período 1880 a 2012, y las proyecciones para finales de siglo oscilan entre $2^{\circ} \mathrm{C}\left(\mathrm{RCP}{ }^{1} 2,6\right)$ y $5^{\circ} \mathrm{C}(\mathrm{RCP} 8,5)$. El promedio anual de la temperatura global siempre ha variado entre períodos fríos y cálidos; sin embargo, es evidente que en las últimas tres décadas se ha producido un aumento progresivo en la temperatura media del planeta, con variaciones importantes a nivel regional, lo que ha provocado cambios en la cantidad, la intensidad, la frecuencia y el tipo de precipitación pluvial (IPCC, 2013).

El aumento de la temperatura media del planeta también ha provocado el calentamiento del océano y el derretimiento de los glaciares. En los últimos 40 años, la temperatura media del océano registra un incremento de $0,44{ }^{\circ} \mathrm{C}$, pues el océano representa un $90 \%$ de la energía acumulada en el planeta. La expansión térmica y la adición del agua dulce proveniente del derretimiento de los glaciares ha provocado una elevación del nivel medio del mar en aproximadamente 0,19 metros, y podría aumentar 
hacia fin del siglo entre 0,26 a 0,98 metros. Aunado a ello, los océanos han absorbido alrededor del 30\% del $\mathrm{CO}_{2}$ emitido, por lo cual se han acidificado. Se estima que el $\mathrm{pH}$ ha disminuido en 0,1 unidades y que en los próximos años podría disminuir hasta en 0,32 unidades (IPCC, 2013).

Todos estos cambios en los sistemas biofísicos del planeta tienen un impacto sobre los ecosistemas (pérdida de hábitats y especies) y los sistemas humanos (salud, transporte, vivienda, negocios. Estos últimos, sobre todo, por la ocurrencia más frecuente y severa de eventos extremos del clima como huracanes, frentes fríos, olas de calor, tornados, inundaciones y sequías (Santiago et al., 2008). Estos fenómenos causan lesiones, enfermedades, defunciones y pérdidas económicas, lo que pone en evidencia y exacerba la desigualdad y vulnerabilidad de las poblaciones humanas, los riesgos construidos cotidianamente y la ausencia de la prevención necesaria.

Para evitar un cambio climático extremo que incremente las consecuencias negativas, el aumento en la temperatura media del planeta debería ser menor a los $2{ }^{\circ} \mathrm{C}$, para ello las emisiones de $\mathrm{CO}_{2}$ acumuladas no deben sobrepasar los 1000 giga toneladas (GtC). Sin embargo, para el 2011 ya se habían emitido más de la mitad de esta cantidad (IPCC, 2013). Por lo tanto, es urgente adoptar medidas para estabilizar las concentraciones de gases de efecto invernadero en la atmósfera. Entre estas medidas, se destacan las estrategias de desarrollo bajo en emisiones (LEDS, por sus siglas en inglés); esto es, reducir emisiones mediante una mayor eficiencia energética en los equipos, evitar construcción de fábricas con emisiones intensivas, invertir en tecnologías limpias y energías alternativas, preservar y potenciar los sumideros y reservorios de GEI (Unfccc, 2009), entre otras. Se trata de disociar el crecimiento económico y el desarrollo social de las emisiones de gases de efecto invernadero, y esto requiere un cambio fundamental del modo de producir, consumir y desechar del sistema económico hegemónico (Organismo Alemán de Cooperación Internacional [GIZ], 2012).

Los Estados de la Convención Marco de las Naciones Unidas sobre el Cambio Climático (Cmnucc), en la reunión Copenhague en el 2009, establecieron compromisos para reducir las emisiones de GEI al menos en un 50\% para 2050 (Programa de Naciones Unidas para el Medio Ambiente [Pnuma], 2014). Sin embargo, aun cuando se consiga esa reducción en las emisiones en comparación con los niveles de 2005, y así limitar el calentamiento climático a $2{ }^{\circ} \mathrm{C}$, los países en desarrollo continuarán enfrentándose a los efectos adversos del cambio climático (Stern, 2006), en consecuencia a la inercia climática. Por lo tanto, para estos países es fundamental emprender acciones de adaptación e incrementar su resiliencia.

Las respuestas de adaptación en el ámbito de la agricultura tendrían que incorporarse como parte de las prácticas agrícolas ya existentes y los conocimientos tradicionales vigentes, en los planes y proyectos para el desarrollo comunitario con enfoque sustentable, y como parte de las políticas sectoriales agrícolas (Organización para la Cooperación y el Desarrollo Económico [OCDE], 2010). Adicionalmente, para fortalecer la resiliencia comunitaria, es decir, fortalecer la capacidad de recuperación de la comunidad a los cada vez más frecuentes y aleatorios desastres ocasionados por la variabilidad climática, es necesario vincular las iniciativas de adaptación a las estrategias de reducción del riesgo de desastres, aunque estas abarquen eventos que no estén relacionados con el cambio climático, como los terremotos o las erupciones volcánicas. Sobre todo, dichas estrategias abordan desastres como inundaciones, sequias y huracanes, es decir, eventos meteorológicos exacerbados por el cambio climático (Oxfam, 2010).

El medio rural enfrenta difíciles desafíos que están interrelacionados, debe adaptarse y aumentar su resiliencia al cambio climático y mitigar sus emisiones de GEI (Organización de las Naciones Unidas para la Alimentación y la Agricultura [FAO], 2016); pero, sobre todo, debe garantizar la seguridad alimentaria y mantener la identidad cultural y territorial de las comunidades. Las estrategias para el logro de dichos desafíos deberán estar basadas en tres principios fundamentales: a) opciones útiles en todo caso, es decir, medidas que generan beneficios significativos aunque no exista un escenario de cambio climático; b) el principio precautorio, que respalda la adopción de medidas protectoras, aun sin contar con evidencias contundentes de los posibles efectos del cambio climático (PNUD, 2012); y c) la participación de todas las partes interesadas en el proceso de adopción de dichas estrategias, la cual es fundamental para la gobernanza, 
aumenta la aplicación eficaz de las estrategias y contribuye a evitar duplicidad de esfuerzos, y permite que sean escuchadas las voces de aquellos más vulnerables en la sociedad (Comisión Económica y Social de Naciones Unidas [Unescap], 2006).

\section{Metodología}

La investigación se realizó con un enfoque estructural exploratorio de tipo interpretativo, basado en la herramienta metodológica de historias de vida (Tinoco \& Bellato, 2006), la cual permite la sistematización y la recomposición focalizada sobre las percepciones y representaciones sociales de una dimensión de interés mediante el relato biográfico de un actor clave de dicha dimensión. En este caso, la dimensión de interés fue los cambios ambientales en el estado de Chiapas, con énfasis en indicadores del cambio climático local.

El relato biográfico es el componente central de la historia de vida, es el relato detallado de la dimensión de interés como un proceso de larga duración mediante la selección de recuerdos, descripción detallada de espacios, eventos coyunturales, datos y fechas. Este debe permitir entrelazar las etapas de vida del informante clave con eventos coyunturales de dicha dimensión. En esta investigación, el relato biográfico se logró por medio de una o más entrevistas semi estructuradas a cada uno de los doce informantes seleccionados, en algunos casos también fueron completadas mediante conversaciones informales y observación participante. El método requiere de plena confianza para la realización de las entrevistas; debe existir un consentimiento informado, secreto profesional y un ambiente propicio, elementos que un método de selección aleatorio no puede garantizar. Por lo tanto, los informantes clave o expertos comunitarios fueron seleccionados bajo el método no estadístico de conveniencia de sitio, pues lo que se buscaba era contar con historias de vida de distintas realidades socioambientales del estado de Chiapas, para lo cual se identificaron hombres y mujeres de mayor edad en comunidades rurales representativas de las distintas regiones biogeográficas del estado. Los municipios considerados en este estudio fueron Amatenango de la Frontera, Amatenango del Valle, Ángel Albino Corzo, Chilón, Francisco León, La Trinitaria, Mazapa de Madero, San Juan Cancuc, Teopisca y Tila.

El análisis de las historias de vida se realizó con un enfoque hermenéutico en las representaciones sociales de los cambios ambientales en el estado de Chiapas. Con este fin, se construyó una matriz de interpretación para contrastar cada caso observado con cinco categorías analíticas: a) antes, b) ahora, c) causas, d) consecuencias y c) responsables. A partir de esta matriz de análisis, se identificaron los núcleos figurativos y se construyó una tipología para la comprensión de las representaciones sociales estudiadas. La validez de la tipología propuesta se verificó mediante la prueba estadística de $\chi^{2}$.

\section{Resultados y discusión}

Las historias de vida resultaron vastas y enriquecedoras para la pregunta de investigación planteada (se puede consultar el trabajo etnográfico íntegro en Santiago, 2015). Con un lenguaje sencillo pero profundo, los expertos comunitarios dieron cuenta de los cambios ambientales en el territorio Chiapaneco en los últimos 100 años, e incluso sugieren algunas alternativas para detener y revertir la degradación ambiental que ellos perciben. En la tabla 1 se presenta el análisis de las mismas. 
TABLA 1.

Matriz de interpretación de las historias de vida (en cursivas se resaltan los núcleos figurativos identificados)

\begin{tabular}{|c|c|c|c|}
\hline $\begin{array}{ll}\text { Categoria } & \text { Caso } \\
\text { de análisis }\end{array}$ & $\begin{array}{l}\text { Ezequiel Chávez Gonzales, } 72 \text { años, } \\
\text { Palestina, Amatenango de la Frontera. }\end{array}$ & $\begin{array}{l}\text { Luisa Gómez Cruz, } 61 \text { años, Amatenango } \\
\text { del Valle. }\end{array}$ & $\begin{array}{l}\text { Miguel Méndez Hernández, } 87 \text { años, La } \\
\text { Paz, Ángel Albino Corzo. }\end{array}$ \\
\hline Antes & $\begin{array}{l}\text { Todo era puro potrero y bosque, con muchos } \\
\text { árboles y animales. Cuando fundamos la } \\
\text { comunidad no habia agua. E1 clima era fresco. }\end{array}$ & $\begin{array}{l}\text { La comunidad tenia un clima miy favorable } \\
\text { para la siembra, ya que la lluvia llegaba justo } \\
\text { a tiempo. }\end{array}$ & $\begin{array}{l}\text { E1 bosque era muy bonito, toda la comunidad } \\
\text { estaba completamente llena de vegetación con } \\
\text { una gran variedad de árboles. E1 clima era } \\
\text { templado, con lluvias en el verano. Los ríos } \\
\text { estaban totalmente limpios y se contaba con } \\
\text { variedad de animales. }\end{array}$ \\
\hline Ahora & $\begin{array}{l}\text { Toda la naturaleza se ha ido acabando. El } \\
\text { calor ha aumentado porque en el dia, después } \\
\text { de las doce en adelante, hay mucho calor, y } \\
\text { en las madrugadas hace más frío. Ahora hay } \\
\text { veces que llueve mucho fuera de tiempo y hay } \\
\text { más viento. }\end{array}$ & $\begin{array}{l}\text { Hoy en día ha bajado la producción de maíz } \\
\text { porque ya no saben bien cómo vendrán las } \\
\text { lluvias. La temporada de llvvia no llega a } \\
\text { tiempo. }\end{array}$ & $\begin{array}{l}\text { El espacio que ocupa la comunidad es muy } \\
\text { grande. Hay escuelas, parque central, casa } \\
\text { ejidal, drenaje, energia eléctrica, agua potable } \\
\text { y carretera pavimentada. Los cerros están } \\
\text { completamente pelones, sin árboles y sin } \\
\text { animales. Hay un atraso en las lluvias y un } \\
\text { clima muy caluroso. }\end{array}$ \\
\hline Causas & $\begin{array}{l}\text { Con el tiempo, empezaron a llegar más } \\
\text { personas al ejido. Empezó a usarse la } \\
\text { motosierra, ya no sólo para construir casas, } \\
\text { sino para vender madera; la contaminación } \\
\text { con los químicos, el uso del fuego en las } \\
\text { parcelas y la cacería. }\end{array}$ & $\begin{array}{l}\text { En la comunidad se talan muchos árboles } \\
\text { porque nos dedicamos a la alfareria y } \\
\text { ocupamos un fogón tradicional. }\end{array}$ & $\begin{array}{l}\text { Cuando empezaron a abrir carreteras me di } \\
\text { cuenta de que estaban destruyendo el bosque } \\
\text { con esas máquinas. Luego de que ya habia } \\
\text { carretera, fueron llegando más personas a la } \\
\text { comunidad y fue creciendo. Han orientado la } \\
\text { producción agrícola en algo comercial } \\
\text { mediante el uso de agroquimicos. }\end{array}$ \\
\hline Consecuencias & $\begin{array}{l}\text { Estos cambios de clima han afectado la } \\
\text { producción de café porque el clima favorece } \\
\text { que ahora haya más roya y broca. Hay veces } \\
\text { que llueve mucho cuando está maduro el café, } \\
\text { y por tanta agua se cae el grano y se pierde la } \\
\text { cosecha. }\end{array}$ & $\begin{array}{l}\text { La quema en horno tradicional consiste en } \\
\text { quemar al aire libre utilizando gran cantidad } \\
\text { de leña. Es en ese momento que nos damos } \\
\text { cuenta de que se está generando } \\
\text { contaminación que daña el aire y el suelo. }\end{array}$ & $\begin{array}{l}\text { E1 drenaje desemboca en el río y se está } \\
\text { contaminando. Los cultivos no desarrollan } \\
\text { bien por falta de agua. E1 rio está bajando su } \\
\text { nivel porque ya no llueve igual, pero también, } \\
\text { de pronto, se desborda por lluvias muy } \\
\text { intensas. }\end{array}$ \\
\hline Responsables & $\begin{array}{l}\text { Pero ¿qué le vamos a hacer? Si asi es la vida. } \\
\text { Todo cambia. }\end{array}$ & $\begin{array}{l}\text { Por eso me pongo a pensar en el mundo que } \\
\text { le estamos dejando a nuestros nietos, y a } \\
\text { veces pienso en dejar la artesania para no } \\
\text { contaminar más. Pero luego pienso que si lo } \\
\text { dejo ¿cómo voy a obtener recursos } \\
\text { económicos para mantener a mi familia? }\end{array}$ & $\begin{array}{l}\text { Estos desastres están ocurriendo porque } \\
\text { estamos acabando con la naturaleza que nos } \\
\text { rodea. Los campesinos jóvenes no saben lo } \\
\text { que significa un árbol o los recursos } \\
\text { naturales. Estos programas fueron impulsados } \\
\text { por el gobierno que sólo ve la cuestión } \\
\text { económica y no vela por el cuidado de la } \\
\text { naturaleza. }\end{array}$ \\
\hline Antes & $\begin{array}{l}\text { E1 lugar era montañoso, la vegetación muy } \\
\text { abundante, por lo tanto habia muchos } \\
\text { animales. E1 aire era muy agradable, no hacia } \\
\text { frío ni mucho calor. En ese tiempo no había } \\
\text { carretera. }\end{array}$ & $\begin{array}{l}\text { Encontramos una extensa vegetación y una } \\
\text { diversidad de animales muy importante. Con } \\
\text { una laguna sagrada que alimenta un río. }\end{array}$ & $\begin{array}{l}\text { Realizábamos dos veces la siembra en el año } \\
\text { porque habia mucho terreno donde sembrar. } \\
\text { Como todo era montaña, pues teniamos que } \\
\text { tumbar árboles grandes de amate. Durante la } \\
\text { producción, había animales que se comían el } \\
\text { maíz, pero como daba en abundancia, nadie } \\
\text { se preocupaba por cuidarlo. Todo lo que se } \\
\text { consumia se obtenia de la milpa. }\end{array}$ \\
\hline Ahora & $\begin{array}{l}\text { E1 ejido ha crecido mucho; ya no se ven } \\
\text { detrás de las casas los armadillos ni los } \\
\text { venados. No se pueden comer los caracoles } \\
\text { del río porque está contaminado. Después de } \\
\text { la muerte de mi esposa, fui dejando poco a } \\
\text { poco de cultivar la milpa, pues ya nadie hace } \\
\text { las tortillas ni el pozol. }\end{array}$ & $\begin{array}{l}\text { E1 grave problema que existe actualmente en } \\
\text { la comunidad es la deforestación. De nada } \\
\text { sirve conservar los conocimientos } \\
\text { tradicionales si se está destruyendo la } \\
\text { naturaleza. }\end{array}$ & $\begin{array}{l}\text { De tanto que el maiz ya no se daba bien, } \\
\text { algunos decidimos rozar nuestros terrenos } \\
\text { para sembrar pastos y meter ganado, porque } \\
\text { pensábamos que nos ayudaria a tener mayores } \\
\text { ingresos económicos. }\end{array}$ \\
\hline Causas & $\begin{array}{l}\text { Se empezó a construir una carretera } \\
\text { provisional, porque en ese tiempo una } \\
\text { compañia ya estaba sacando madera de la } \\
\text { selva. Cuando se retiró la compañia maderera, } \\
\text { empezaron a llegar muchos chicleros a sacar } \\
\text { chicle de la corteza de los árboles, pero ya no } \\
\text { habia mucho que aprovechar. }\end{array}$ & $\begin{array}{l}\text { Debido a la construcción de casas, porque } \\
\text { cada año la población está aumentando, y } \\
\text { también debido a los incendios forestales por } \\
\text { descuidos en las quemas para la preparación } \\
\text { de las parcelas agrícolas. Otro problema fue el } \\
\text { uso de agroquímicos en la producción de café. }\end{array}$ & $\begin{array}{l}\text { Cuando se abrió la carretera y llegó la energía } \\
\text { eléctrica, la gente empezó a salir a otros } \\
\text { lugares, conocieron otras cosas y formas de } \\
\text { cómo trabajar la tierra, entonces empezaron a } \\
\text { saber de los productos químicos. A1 principio } \\
\text { si se lograba sacar una buena cantidad, pero } \\
\text { después de algunos años, ya no. }\end{array}$ \\
\hline Consecuencias & $\begin{array}{l}\text { Mis hijos siembran maíz y palma de aceite, } \\
\text { otros lo convierten en potrero. Yo todavia } \\
\text { tengo mi potrero y una hectárea de palma de } \\
\text { aceite. El clima ha cambiado, ahora en } \\
\text { diciembre hace mucho frío, en febrero ya } \\
\text { llueve más, incluso algunos ya siembran hasta } \\
\text { junio, porque se tiene que esperar la lluvia. } \\
\text { En temporada de calor el sol es más fuerte, el } \\
\text { aire más caliente y la mayoría de los arroyos } \\
\text { se secan y tienen mal olor, pero en temporada } \\
\text { de lluvias los ríos crecen demasiado. }\end{array}$ & $\begin{array}{l}\text { Esto ha provocado que muchos animales } \\
\text { mueran o se alejen, también ha afectado la } \\
\text { fertilidad del suelo. La destrucción de las } \\
\text { montañas hace que no llueva, y la agricultura, } \\
\text { que es nuestro principal sustento, depende de } \\
\text { la lluvia y a veces no logramos producir lo } \\
\text { suficiente, nos vemos obligados a comprar. }\end{array}$ & $\begin{array}{l}\text { Hay meses de puro calor, como marzo, abril } \\
\text { y mayo, también a veces llueve por semanas } \\
\text { como si fuera temporada de lluvias; y en la } \\
\text { temporada de lluvias, como en agosto, } \\
\text { septiembre y octubre, llueve muy poco. Ya no } \\
\text { llueve como antes y no hacia tanto frio en } \\
\text { enero. }\end{array}$ \\
\hline Responsables & $\begin{array}{l}\text { Los que viven más al norte tiran basuras en el } \\
\text { río. Hace como diez años empezaron a dar } \\
\text { apoyos económicos para la siembra de palma. }\end{array}$ & $\begin{array}{l}\text { No dañar a la madre tierra, porque con el paso } \\
\text { del tiempo los perjudicados seremos nosotros, } \\
\text { los seres humanos. }\end{array}$ & $\begin{array}{l}\text { Se ha perdido la fe en la agricultura porque no } \\
\text { siempre se logra tener buena producción. Los } \\
\text { jóvenes ya no saben de la agricultura. }\end{array}$ \\
\hline
\end{tabular}

Fuente: elaboración propia con base en Santiago (2015). 
TABLA 1. (cont.)

Matriz de interpretación de las historias de vida (en cursivas se resaltan los núcleos figurativos identificados)

\begin{tabular}{|c|c|c|c|}
\hline $\begin{array}{ll}\begin{array}{l}\text { Categoria } \\
\text { de análisis }\end{array} & \text { Caso } \\
\end{array}$ & $\begin{array}{l}\text { Luisa Calmo Matías, } 70 \text { años, San Antonio } \\
\text { Tzalani, La Trinitaria. }\end{array}$ & María, 74 años, Mazapa de Madero. & $\begin{array}{l}\text { Pedro Martínez Martínez, } 75 \text { años, } \\
\text { Guadalupe la Providencia, Teopisca. }\end{array}$ \\
\hline Antes & $\begin{array}{l}\text { Recuerdo que sembrábamos sin utilizar } \\
\text { fertilizantes químicos, y como no habia tanta } \\
\text { gente, el terreno era más limpio y daba más la } \\
\text { siembra. Había mucho terreno para sembrar, } \\
\text { las lluvias eran exactas y sabiamos en qué } \\
\text { tiempo sembrar. Se daba bien la cosecha y } \\
\text { habia más animales, árboles y plantas. }\end{array}$ & $\begin{array}{l}\text { Habia mucha montaña, rio, árboles y } \\
\text { animales. No habia carros. Como } \\
\text { trabajábamos bien la tierra, habia mucha } \\
\text { cosecha. La gente trabajaba mucho y pedian } \\
\text { para que lloviera. Teniamos buenas cosechas } \\
\text { porque seguiamos la luna y llovia suficiente. }\end{array}$ & $\begin{array}{l}\text { Sacábamos por tonelada maíz y frijol. En la } \\
\text { comunidad no habia mucha gente ni casas, } \\
\text { había muchos árboles grandes en la montaña } \\
\text { y hongos para comer, abundaban. Habia una } \\
\text { poza donde se juntaba el agua, bien clara y no } \\
\text { se secaba rápido, pues llovia casi todo el año } \\
\text { y habia menos calor. }\end{array}$ \\
\hline Ahora & $\begin{array}{l}\text { He observado que el clima ha cambiado } \\
\text { mucho, a veces llueve aunque no estemos en } \\
\text { época de lluvia. El clima se ha vuelto muy } \\
\text { seco. }\end{array}$ & $\begin{array}{l}\text { La tierra tiene mucho pasto y si la siembran } \\
\text { usan puro abono químico. Llueve menos y ya } \\
\text { no está húmeda la tierra. Ya no hay montaña. }\end{array}$ & $\begin{array}{l}\text { La milpa se fumiga con líquidos y un poco de } \\
\text { fertilizante para que crezca. Se acabó el } \\
\text { bosque y los animales de monte también. } \\
\text { Empezó fuerte el calor y a dejar de llover en } \\
\text { abril. La gente ya no halla dónde tirar su } \\
\text { basura y la deja donde sea. }\end{array}$ \\
\hline Causas & $\begin{array}{l}\text { La población ha aumentado la tala de árboles. } \\
\text { E1 aire ya no es natural, sino que contiene } \\
\text { muchos contaminantes por utilizar tantos } \\
\text { químicos. }\end{array}$ & $\begin{array}{l}\text { Ya se perdieron todas las costumbres. Ya no } \\
\text { trabajan bien la tierra. Tiraron muchos } \\
\text { árboles por la madera para hacer casas y por } \\
\text { la carretera. }\end{array}$ & $\begin{array}{l}\text { Mi papá nos enseñó a producir carbón, por } \\
\text { eso ya se acabaron los árboles. La gente de } \\
\text { fuera mataba los animales y a veces nosotros } \\
\text { mismos los cazábamos para comer. }\end{array}$ \\
\hline Consecuencias & $\begin{array}{l}\text { La producción ha disminuido y le tenemos } \\
\text { que poner más químicos. No hay leña por la } \\
\text { tala de árboles. Cuando llueve en exceso, se } \\
\text { deslava nuestra siembra o se pudre; o cuando } \\
\text { hay mucho calor, la siembra se seca y no da } \\
\text { fruto. Cuando utilizamos químicos en los } \\
\text { cultivos, el líquido sube al cielo y contamina } \\
\text { el aire, o queda en el suelo y va contaminando } \\
\text { los ríos y la tierra misma. No sólo afecta } \\
\text { nuestros cultivos, sino también a los animales } \\
\text { domésticos y silvestres. }\end{array}$ & $\begin{array}{l}\text { Ya no da bien la milpa, ya no alcanza. Mis } \\
\text { hijos ahora salen a trabajar para conseguir } \\
\text { dinero. Ya no hay leña. Se pudre la cosecha, } \\
\text { se seca la milpa. Tampoco lloran en la } \\
\text { montaña los lobos. Si no hay tierra, nos } \\
\text { morimos todos. }\end{array}$ & $\begin{array}{l}\text { Ahora se seca rápido la poza por el calor que } \\
\text { ya está fuerte y el agua se pone verde y huele } \\
\text { muy mal. Por eso ahora dan enfermedades } \\
\text { como diarrea, calentura y otros que no se } \\
\text { curan con hierbas. Dejamos de comer verdura } \\
\text { de la milpa y empezamos a comprarla en el } \\
\text { mercado. Las vacas y los chivos ya no } \\
\text { encuentran su pastura porque como no llueve } \\
\text { rápido, tarda en retoñar el pasto. E1 liquido } \\
\text { que se le pone a la milpa es malo para } \\
\text { nosotros y más para nuestras esposas. }\end{array}$ \\
\hline Responsables & $\begin{array}{l}\text { Nosotros contaminamos el ambiente, lo } \\
\text { maltratamos usando químicos y quemando la } \\
\text { basura o tirándola en las calles. E1 clima va } \\
\text { cambiando por el desastre que hacemos con } \\
\text { nuestro planeta. }\end{array}$ & $\begin{array}{l}\text { Yo me siento triste porque cortaron los } \\
\text { arboles. Ahora estoy aconsejando a mis hijos } \\
\text { para que aprendan a trabajar bien la tierra. }\end{array}$ & $\begin{array}{l}\text { Sabemos que somos nosotros quienes } \\
\text { maltratamos la tierra y por eso no llueve. } \\
\text { Empezamos a ponerle un poco de fertilizante } \\
\text { y a matar el monte con líquido, lo empezamos } \\
\text { a usar porque nos contaron que asi debe más } \\
\text { la milpa. Pero vimos también que cada vez } \\
\text { hacía falta más dinero para comprar los } \\
\text { fertilizantes y líquidos. }\end{array}$ \\
\hline Antes & $\begin{array}{l}\text { La cosecha era muy buena. La luna era como } \\
\text { nuestro calendario, ella nos decía cuándo } \\
\text { sembrar y cuándo nos mandaría la lluvia. }\end{array}$ & $\begin{array}{l}\text { La cosecha de milpa y frijol alcanzaba para } \\
\text { que la familia se alimentara durante un año, } \\
\text { incluyendo la alimentación de sus animales de } \\
\text { traspatio, pues anteriormente se cosechaba } \\
\text { bastante y crecian bien las plantas de la milpa } \\
\text { sin necesidad de productos químicos. Las } \\
\text { plantas de la milpa crecian más altas y daban } \\
\text { elotes más grandes. }\end{array}$ & $\begin{array}{l}\text { E1 clima era caluroso en los meses de marzo a } \\
\text { mayo, los meses de julio a octubre eran los } \\
\text { más lluviosos. Podiamos ver con facilidad } \\
\text { animales silvestres debido a que habia } \\
\text { muchas montañas cercanas al pueblo. Se } \\
\text { podia ver, en gran cantidad, los árboles de } \\
\text { cedro y caoba. Los ríos Chijtieja y el arroyo } \\
\text { E1 Sumidero tenian aguas limpias. }\end{array}$ \\
\hline Ahora & $\begin{array}{l}\text { La tierra ya no nos da nuestras mazorcas } \\
\text { grandes ni un frijolar frondoso. Los cerros } \\
\text { están todos pelones porque nuestra madre } \\
\text { tierra ya no tiene fertilidad. }\end{array}$ & $\begin{array}{l}\text { E1 rendimiento del maiz ya es mucho menor a } \\
\text { pesar del uso de agroquimicos. Ahora crecen } \\
\text { más delgaditas y las mazorcas son y } \\
\text { pequeñas. }\end{array}$ & $\begin{array}{l}\text { Los ríos ahora están contaminados porque se } \\
\text { descargan las aguas negras. Los animales } \\
\text { silvestres se han refugiado en cerros donde el } \\
\text { hombre aún no ha llegado. }\end{array}$ \\
\hline Causas & $\begin{array}{l}\text { Si fuera joven, ya no echaria más líquido a la } \\
\text { milpa, porque pienso que ese es el problema } \\
\text { de que la tierra ya no dé, y que por eso se } \\
\text { calienta mucho el clima. Yo considero que el } \\
\text { uso de los liquidos en la milpa daña mucho a } \\
\text { la santa tierra y bastante al clima. }\end{array}$ & $\begin{array}{l}\text { Llegaron los productos quimicos que, en la } \\
\text { actualidad, se siguen usando ya que, si no se } \\
\text { utilizan estos productos, ya no da buena } \\
\text { cosecha, y la gente ya se acostumbró a } \\
\text { usarlos. Es porque ya no se cuida bien a la } \\
\text { madre tierra. }\end{array}$ & $\begin{array}{l}\text { En la década de los ochenta los campesinos } \\
\text { empezaron a usar productos químicos como } \\
\text { los fertilizantes, los insecticidas y los } \\
\text { herbicidas. Otra causa es el crecimiento de la } \\
\text { población, que ha afectado con la tala de } \\
\text { árboles por la construcción de casas. }\end{array}$ \\
\hline Consecuencias & $\begin{array}{l}\text { Ya no llueve en su tiempo, hace más calor y } \\
\text { el viento corre más fuerte. Ahora hace mucho } \\
\text { frio en las tardes y mucho calor en el dia. Ya } \\
\text { no llueve y la milpa no se da bien. }\end{array}$ & $\begin{array}{l}\text { Ahora hace más frio y llueve más. Poco a } \\
\text { poco se contamina el medio ambiente y } \\
\text { después ya no crecerá nada en esta tierra. } \\
\text { Muchos ahora están migrando a otros estados } \\
\text { para conseguir algo de dinero. }\end{array}$ & $\begin{array}{l}\text { Ha aumentado el calor y ya no sabemos } \\
\text { cuándo va a empezar la temporada de llavias, } \\
\text { porque a veces se atrasa o se adelanta. Las } \\
\text { lluvias de octubre y noviembre son más } \\
\text { fuertes por el paso de ciclones muy seguidos. }\end{array}$ \\
\hline Responsables & $\begin{array}{l}\text { E1 gobernador del Estado estaba regalando } \\
\text { paquetes de bombas para fumigar, } \\
\text { fertilizantes y líquidos químicos para mejorar } \\
\text { nuestra cosecha. Nos olvidamos del azadón y } \\
\text { la coa, pensamos que la tierra y la luna } \\
\text { estaban enojadas con nosotros por lo que les } \\
\text { estábamos dando de comer veneno, por } \\
\text { nuestra ignorancia de humanos. Muchos } \\
\text { quieren poner abonos orgánicos, cuando } \\
\text { desde antes los usábamos y fue el gobierno el } \\
\text { que promovió el uso de los quimicos, y } \\
\text { nosotros, por torpes, lo aceptamos. }\end{array}$ & $\begin{array}{l}\text { La gente de la comunidad que utiliza los } \\
\text { productos químicos y ya no reza antes de } \\
\text { sembrar como lo hacian los abuelos. }\end{array}$ & $\begin{array}{l}\text { Nuestra naturaleza está desapareciendo por } \\
\text { todo lo que hacemos las personas, por el } \\
\text { consumo excesivo, la tala de arboles y la } \\
\text { destrucción del hábitat de los animales. } \\
\text { Algunos tienen más culpa que otros, pero } \\
\text { todos alguna vez hemos hecho alguna cosa } \\
\text { que daña a la naturaleza. Pero si la afectamos } \\
\text { a ella también nos afectamos a nosotros } \\
\text { mismos y a nuestra familia. }\end{array}$ \\
\hline
\end{tabular}

Fuente: elaboración propia con base en Santiago (2015). 
Del análisis de la matriz de interpretación de las historias de vida, se identificaron 11 núcleos figurativos para las cinco categorías de análisis. Es claro que en la narrativa de las historias de vida hay quienes recuerdan el pasado en función de la conservación de los bosques, y quienes recuerdan el pasado en función de las abundantes cosechas en sus parcelas. Hay tres escenarios sobre el cambio en el clima: dos aluden al aumento de la temperatura, pero se diferencian porque uno considera que las lluvias han disminuido, mientras que el otro considera que han sufrido un atraso en el año, y sólo un caso considera una disminución de la temperatura con aumento de las lluvias. Las causas de estos cambios ambientales las atribuyen a la deforestación, al uso de agroquímicos y al aumento de la población. Al señalar responsabilidades, varios la asumen como tal y otros señalan a terceros. La tabla 2 resume los núcleos figurativos identificados y reporta las frecuencias absolutas.

TABLA 2.

Núcleos figurativos, entre paréntesis se reporta la frecuencia absoluta observada

\begin{tabular}{|c|c|c|c|}
\hline Categoría de análisis & \multicolumn{3}{|c|}{ Núcleos figurativos } \\
\hline Antes & $\begin{array}{l}\text { Bosque conservado, } \\
\text { abundante fauna y ríos } \\
\text { limpios (6). }\end{array}$ & $\begin{array}{c}\text { Abundante cosecha sin } \\
\text { necesidad de } \\
\text { agroquímicos (6). }\end{array}$ & \\
\hline Ahora o Consecuencia & $\begin{array}{c}\text { Aumento de la } \\
\text { temperatura y disminución } \\
\text { de lluvias (5). }\end{array}$ & $\begin{array}{c}\text { Aumento de la } \\
\text { temperatura y atraso en } \\
\text { las lluvias (6). }\end{array}$ & $\begin{array}{c}\text { Disminución de la } \\
\text { temperatura y aumento } \\
\text { de lluvias (1). }\end{array}$ \\
\hline Causa & Deforestación (4). & Agroquímicos (5). & $\begin{array}{l}\text { Aumento de población } \\
\text { (3). }\end{array}$ \\
\hline Responsable & Nosotros (6). & Otros (4). & Gobierno (2). \\
\hline
\end{tabular}


Con base en los núcleos figurativos identificados, se construyó una tipología de las representaciones sociales del cambio climático global en el medio rural del estado de Chiapas, y se verificó la validez de dicha tipología mediante la prueba estadística de Ji cuadrada $\left(\chi^{2}\right)$. Se ocupó el valor crítico de 4,61 correspondiente a una probabilidad de error menor al 10\% $(\mathrm{P}<0,1)$ con 2 grados de libertad, para descartar la hipótesis nula de independencia de la clasificación o tipología propuesta (tabla 3).

Reconocer dos tipos de representaciones sociales del cambio climático global en el medio rural del estado de Chiapas, tiene sentido con base en la causa que asocian a dicho cambio, y estadísticamente resultó poco significativo $\left(\chi^{2}=4,8\right)$. El 50\% de los sabios comunitarios entrevistados tienen un discurso asociado al estado de conservación de los bosques y su fauna asociada, y el 50\% de ellos asocian el deterioro de los bosques y la pérdida de fauna al aumento de la población y lo que ello representa en demanda de espacio para urbanización, consumo y desecho. En efecto, el estado de Chiapas presenta un ritmo de crecimiento demográfico acelerado, al pasar de 400 mil habitantes en 1900, a poco más de 4,7 millones de personas, en 2010 (Instituto Nacional de Estadística y Geografía [Inegi], 2013), y se estima un poco más de 6 millones de habitantes para el 2030 (Consejo Nacional de Población [Conapo], 2010). Este crecimiento demográfico está correlacionado con los datos registrados para el periodo comprendido entre 1990 y 2007 de una tasa anual de cambio en la cobertura forestal de 5,4\%, una de las más altas registradas en el mundo (Morales, 2009).

TABLA 3.

Tipología propuesta de representaciones sociales del cambio climático global en el medio rural del estado de Chiapas.

\begin{tabular}{|c|c|c|c|}
\hline Núcleo Figurativo & Conservacionistas & Productivistas & Total \\
\hline Aumento de la temperatura y disminución de lluvias & $2(2,5)$ & $3(2,5)$ & 5 \\
\hline Aumento de la temperatura y atraso en las lluvias & $4(3)$ & $2(3)$ & 6 \\
\hline Disminución de la temperatura y aumento de lluvias & $0(0,5)$ & $1(0,5)$ & 1 \\
\hline $\begin{array}{c}\text { Total } \\
\chi^{2} \\
\end{array}$ & 6 & 6 & $\begin{array}{l}12 \\
1,9 \\
\end{array}$ \\
\hline Deforestación & $2(2)$ & $2(2)$ & 4 \\
\hline Agroquímicos & $1(2,5)$ & $4(2,5)$ & 4 \\
\hline Aumento de población & $3(1,5)$ & $0(1,5)$ & 4 \\
\hline $\begin{array}{c}\text { Total } \\
\chi^{2}\end{array}$ & 6 & 6 & $\begin{array}{c}12 \\
4,8^{*}\end{array}$ \\
\hline Nosotros & $3(3)$ & $3(3)$ & 6 \\
\hline Otros & $2(2)$ & $2(2)$ & 4 \\
\hline Gobierno & $1(1)$ & $1(1)$ & 2 \\
\hline $\begin{array}{c}\text { Total } \\
\chi^{2}\end{array}$ & 6 & 6 & $\begin{array}{l}12 \\
0,0\end{array}$ \\
\hline
\end{tabular}

Entre paréntesis se reportan los valores esperados de acuerdo a la prueba estadística de Ji cuadrada. El asterisco $\left({ }^{*}\right)$ señala diferencias estadísticamente significativas con base en el valor crítico $\left(\chi^{2} 0.10,2=4,61\right)$ Fuente: elaboración propia

En tanto que el otro $50 \%$ de los entrevistados tiene un discurso asociado a la producción agrícola, recuerdan que en antaño las cosechas eran abundantes y no se requerían insumos externos para producirlas. El $67 \%$ de ellos asocia el deterioro ambiental actual al alto uso de agroquímicos en la producción agropecuaria. En efecto, a partir de la década de los setenta del siglo pasado, las agencias internacionales de desarrollo, por medio de las instituciones gubernamentales, promovieron los paquetes tecnológicos y su ideología del progreso con base en el uso de energía fósil y mineral a partir de la maquinaria agrícola, las semillas mejoradas, los fertilizantes e insecticidas químicos (Toledo, 2000). Se ha documentado ampliamente que este sistema productivo transforma a los campesinos en dependientes de insumos de alto costo, insumos que pueden tener serios efectos sobre la salud de productores y consumidores (Altieri, Rosset, \& Thrupp, 2001; Montoya, Bello, 
Parra, \& Mariaca, 2003; Seefoó, 2005). Además, también está provocando la desaparición del conocimiento tradicional en el manejo de los recursos naturales, un desequilibrio ambiental y la desarticulación de su anterior sistema productivo (Boege, 2008).

Con respecto a quien responsabilizan del deterioro ambiental y del cambio climático, no hay diferencias entre las dos tipologías. En general, el 50\% de los entrevistados se asumen como responsables y el otro 50\% culpa a otros, ya sea al gobierno, por promover paquetes tecnológicos de alto uso de insumos industriales, o simplemente a otras personas que, con sus acciones, han deteriorado el ambiente.

Finalmente, en cuanto a la dimensión de mayor interés en este estudio, sobre las representaciones sociales del cambio climático, en general hay un fuerte conceso ( $92 \%$ de los entrevistados) sobre la percepción de un aumento en la temperatura promedio anual y cambios en el patrón de lluvias. El 50\% considera que la temporada de lluvias ha sufrido un atraso en el período de ocurrencia, y el $42 \%$ de los entrevistados percibe una disminución en las precipitaciones. Esto concuerda con los escenarios modelados para el estado de Chiapas, de acuerdo a la plataforma de resultados ofrecida por el Instituto Nacional de Ecología y Cambio Climático (Inecc), sobre la actualización de escenarios de cambio climático para un futuro cercano (2015 - 2039) y con trayectorias de concentraciones representativas (RCP) 4,5, el cual es un escenario de emisiones moderado, con una radiación global de energía de 4,5 $\mathrm{Wm}^{-2}$ estable después del 2100 , y concentraciones de 650 p. p. m. de $\mathrm{CO}_{2}$ (Moss et al., 2010). Además, con la base de datos climáticos de la Unidad de Investigación Climática (CRU, por sus siglas en inglés) versión TS3.1 (CRU, 2012), se proyecta un aumento de la temperatura media anual entre los 0,8 y $1,6^{\circ} \mathrm{C}$, y una reducción en la precipitación entre los $-0,05$ y $-0,35 \mathrm{~mm} / \mathrm{d}$, lo que significa años cada vez más calurosos y más secos, con eventos meteorológicos extremos más intensos (Santiago, 2015).

\section{Conclusiones}

Los hallazgos documentados en este artículo son las representaciones sociales de mujeres y hombres de las comunidades rurales sobre los cambios ambientales en Chiapas, a partir del detallado relato de su entorno a lo largo de su vida. Estas percepciones ambientales de la gente "común" (agricultores, campesinos, amas de casa en el medio rural, ancianos y ancianas), verdaderos expertos del medio ambiente, no distan de lo documentado desde la visión de la ciencia occidental.

Los sabios comunitarios dan testimonio de la gran diversidad biológica y agrícola del territorio Chiapaneco a principios del siglo pasado, y cómo se fue perdiendo a raíz del incentivo de las políticas de "modernización del campo" mediante paquetes tecnológicos que promueven los monocultivos a base de fertilizantes químicos, insecticidas, herbicidas y fungicidas. Los cultivos, sustento de la familia, se convirtieron en productos para un mercado cada vez más demandante debido al crecimiento poblacional. En ese sentido, las representaciones del deterioro ambiental y del cambio climático se basan en un sentimiento de culpa en una dimensión individual, pero sobre todo en el colectivo por la adopción de dichos paquetes tecnológicos para la producción agropecuaria. Desde estas representaciones sociales documentadas, el cambio climático es un hecho indiscutible.

La tarea que queda pendiente por atender es la sistematización del conocimiento experto sobre respuestas locales a los efectos asociados a los cambios ambientales en las comunidades rurales de Chiapas, para diseñar una agenda local de alternativas de adaptación, mitigación y resiliencia a los efectos asociados del cambio climático global. Esta agenda debe contribuir a fortalecer procesos organizativos y educativos que reactiven la soberanía alimentaria, las economías locales, el manejo de los bienes comunes y la defensa del territorio, a partir de potenciar y recrear las capacidades y los saberes locales de producción, y el procesamiento y conservación de alimentos en el ámbito familiar, mediante la agroecología y el manejo comunitario del agua. 


\section{Notas}

Este artículo es parte de los resultados del proyecto Percepciones y respuestas locales al Cambio Climático Global. Recibió apoyo de los programas Profocie-SEP, SEI-Cocytech y SNI-Conacyt, y fue ejecutado en el periodo 2015-2017.

\section{Referencias}

Abric, J. (2001). Prácticas sociales y representaciones. México: Ediciones Coyoacán, S. A. de C. V.

Altieri, M., Rosset, P., y Thrupp L. (2001). Una visión de la alimentación, la agricultura y el medio ambiente en el año 2020. Recuperado el 25/06/2001, de http://www.eter.ws/.

Boege, E. (2008). El patrimonio biocultural de los pueblos indígenas de México. México: INAH. CDI.

Consejo Nacional de Población (Conapo). (2010). Chiapas: Proyecciones de población de CONAPO 2010-2030. Recuperado el 30/01/2014, de http://www.ceieg.chiapas.gob.mx/home/.

Unidad de Investigación Climática (CRU). (2012). Base de datos climáticos CRU versión TS3.1. Escuela de Ciencias Ambientales de la Universidad del Anglia Oriental. Recuperado el 20/08/2012, de http://www.cru.uea.ac.uk.

Organización de las Naciones Unidas para la Alimentación y la Agricultura (FAO). (2016). Cambio climático y seguridad alimentaria y nutricional en América Latina y el Caribe. Orientaciones de política. Recuperado el 18/09/2016, de http://www.fao.org/fileadmin/user_upload/rlc/docs/Cambioclimatico.pdf.

Figueredo, J. (2012). Racionalidad y subjetividad ambiental. Una apuesta ante el cambio climático. Caminos Revista Cubana de Pensamiento Socioteológico. Recuperado el 12/02/2014, de http://revista.ecaminos.org/article/racio nalidad-y-subjetividad-ambiental-una-apuesta- $\%$

Gastrón, L. (2003). Una mirada de género en las representaciones sociales de la vejez. La Aljaba, 8, 177-192.

Organismo Alemán de Cooperación Internacional (GIZ). (2012). Factsheet: Low Emission Development Strategies (LEDS) Global Partnership. Recuperado el 15/08/2015, de http://www.nrel.gov/docs/gen/fy12/53458.pdf

Ibáñez, T. (1994). Psicología social construccionista. México: Universidad de Guadalajara.

Instituto Nacional de Estadística y Geografía (Inegi). (2013). México en cifras. Recuperado el 12/02/2014, de http:/ /www3.inegi.org.mx/sistemas/mexicocifras/.

Panel Intergubernamental sobre Cambio Climático (IPCC). (2013). Cambio Climático 2013: Bases físicas. Resumen para responsables de politicas. Recuperado de https://www.ipcc.ch/pdf/assessment-report/ar5/wg1/WG1AR5 _SPM_brochure_es.pdf

Knapp, E., Suárez, M. y Mesa, M. (2003). Aspectos teóricos y epistemológicos de la categoría Representación Social. Revista Cubana de Psicologia, 20(1), 23-34.

Meira, P. (2013). Problemas ambientales globales y educación ambiental: Una aproximación desde las representaciones sociales del cambio climático. Revista Integra Educativa, 6(3), 29-64.

Montoya, G., Bello, E., Parra, M. y Mariaca, R. (2003). La frontera olvidada entre Chiapas y Quintana Roo. México: Biblioteca popular de Chiapas.

Morales, M. (2009). Plan de acción de cambio climático para el estado de Chiapas. Conservación Internacional México, A. C.

Moss, R., Edmons, J., Hibbard, K., Manning, M., Rose, S., Vuuren, D., ... Wilbanks, T. (2010). The next generation of scenarios for climate change research and assessment. Nature, 463, 747-755. https://doi.org/10.1038/ nature 08823

Moscovici, S. (1979). Elpsicoanálisis, su imagen y su público. Buenos Aires, Argentina: Editorial Huemul S. A. http:// dx.doi.org/10.1037/h0067186.

Organización para la Cooperación y el Desarrollo Económico (OCDE). (2010). Integración de la Adaptación en la Cooperación para el Desarrollo: Guía sobre Politicas. https://doi.org/10.1787/9789264088320-es. 
Organización Meteorológica Mundial (OMM). (2014). Boletín de la OMM sobre los gases de efecto invernadero No. 10. Recuperado de https://www.wmo.int/pages/documents/ghg-bulletin_10_es.pdf.

Oxfam International. (2010). Climate Change Adaptation. Enabling people living in poverty to adapt. Oxfam Research Report. Recuperado de http://earthscience.bcsdk12.org/earthscienceiscool/media/climatechange/documents/ 2010\%20Climate_change_adaptation.pdf.

Programa de Naciones Unidas para el Desarrollo (PNUD). (2012). Multi-Stakeholder Decision-Making. Recuperado el 19/10/2016, de http://www.undp.org/content/dam/undp/library/Environment\%20and\%20Energy/Clim ate\%20Strategies/Multi-stakeholder\%20Decision-Making_Sept\%202012.pdf

Programa de Naciones Unidas para el Medio Ambiente (Pnuma). (2014). The Emissions Gap Report 2014. Nairobi: United Nations Environment Program (UNEP). Recuperado el 23/12/2016, de http://www.unep.org/emissi onsgapreport2014/-.

Ramírez-Vázquez, Y. y González-Gaudiano, E. (2016). Representaciones sociales del cambio climático en estudiantes de dos universidades veracruzanas. CPU-e Revista de investigación educativa, 22, 1-27.

Rizo, M. y Romeu V. (2006). Cultura y comunicación, Aproximaciones conceptuales. e-Compós, (6), 1-19.

Santiago, J., López, M., y López, S. (2008). Tendencias del cambio climático global y los eventos extremos asociados. RaXimbai, 4(3), 625-633.

Santiago, J. (Coord.). (2015). Cambios Ambientales en Chiapas. Doce testimonios del medio rural. México: UNICH Ediciones Navarra.

Seefoó, J. (2005). La calidad es nuestra, la intoxicación ... ide usted! México: El Colegio de Michoacán.

Stern, N. (2006). Stern Review: The Economics of Climate Change. Executive Summary. Recuperado el 26/03/2016, de http://www.wwf.se/source.php/1169157/Stern\%20Report_Exec\%20Summary.pdf.

Tinoco, R. y Bellato L. (2006). Representaciones sociales de la pobreza en Chiapas. México: Ecosur.

Toledo, V. (2000). La paz en Chiapas. Ecología, luchas indígenas y modernidad alternativa. México: Ediciones Quinto Sol.

United Nations Framework Convention on Climate Change (Unfccc). (2009). Fact sheet: The need for mitigation. Recuperado de https://unfccc.int/files/press/backgrounders/application/pdf/press_factsh_mitigation.pdf.

Comisión Económica y Social de Naciones Unidas (Unescap). (2006). Good governance and the Millennium Development Goals. Recuperado el 3/06/2016) de http://www.unescap.org/sites/default/files/Governance.pdf.

[1] Trayectorias de Concentraciones Representativas ( $\mathrm{RCP}$ por sus siglas en inglés), son escenarios de emisiones con base en una radiación global de energía determinada y concentraciones particulares de $\mathrm{CO}_{2}$ (Moss et al., 2010).

\section{Licencia Creative Commons CC BY 4.0}

Cómo citar este artículo: Santiago, J. A., Horita, L. H., y Sulvarán, J. L. (2018). El cambio climático y sus representaciones sociales en el medio rural de Chiapas, México. Ambiente y Desarrollo, 22(42), 1-12. https://doi.org/10.11144/Javeriana.ayd22-42.ccrs 